\title{
Density Waves in a System of Non-Interacting Particles
}

\author{
E. J. Kolmes ${ }^{\mathrm{a}}$, V. I. Geyko ${ }^{\mathrm{a}}$, N. J. Fisch ${ }^{\mathrm{a}}$
}

${ }^{a}$ Department of Astrophysical Sciences, Princeton University, Princeton, New Jersey 08544, USA

\begin{abstract}
An ensemble of non-interacting bouncing balls being acted on by a constant gravitational force, starting at rest from a uniform density distribution, will develop a structure of sharply peaked density waves. We describe these waves by computing the density profile of such a system analytically, and we find that the analytical results are in good agreement with numerical findings. We suggest that in a real system, these density waves could be used to produce measurements of the strength of a gravitational field.
\end{abstract}

Keywords: Dynamical systems, emergent phenomena, nonlinear dynamics, phase space evolution, gravity measurements

\section{Introduction}

Systems of elastically or inelastically bouncing balls have been a subject of interest in a number of different fields of physics. Researchers have used systems involving bouncing balls to study the behavior of dynamical systems, and especially the emergence and characteristics of chaos. Different variations of these systems are often complex enough to demonstrate physically interesting behavior while still being simple enough to be analytically tractable. One well-studied variant involves an oscillating or vibrating lower boundary with which the balls can exchange energy [1, 2]. Such systems are often used as a model for Fermi acceleration [3-7]. A number of authors have studied the dynamical effects of collisions with differing degrees of elasticity or inelasticity [8, 9]. Related systems have also been studied in the context of adding gravity to the classical billiard problem [10].

When used as a vehicle with which to better understand nonlinear dynamical systems, models of this kind can potentially be useful for understanding the behavior of a range of systems ranging from population biology to cryptography [11]. However, bouncing-ball models also be useful in more direct ways. For instance, the complexities associated with bouncing dynamics may help to explain chaotic behavior in structures that contain pin joints with internal degrees of freedom [12].

Complex density wave structures can arise in systems without any inter-particle interactions, under the right conditions. One particularly simple example is a onedimensional system of particles in a linear gravitational potential $V(x)=m g x$ with boundary conditions so that the particles bounce elastically at $x=0$ (in this paper, the boundary will not vibrate or oscillate). If $N$ particles begin

Email address: ekolmes@princeton.edu (E. J. Kolmes) at rest, uniformly distributed between $x=0$ and $x=L$, we start to see a characteristic pattern of high-density pulses immediately. Specifically, we see a series of sawtooth-like structures that propagate from small $x$ to large $x$ before disappearing at $x=L$. As time passes, these saw-toothed waves become increasingly frequent and thinner. Figure 1 shows a series of images of these saw-toothed patterns.

We will start by deriving an analytical expression for the density distribution of the particles in this system at an arbitrary time. We will observe in this expression the presence of saw-toothed wave structures that have all of the characteristics that we expect, and that are in good agreement with the results of numerical simulations, as shown in Figure 2. We will also discuss possible applications of this phenomenon.

\section{Partitioning Parameter Space}

For a particle that starts at rest at a position $x_{0}$, it takes a total interval of time $\Delta t_{f}=\sqrt{2 x_{0} / g}$ to fall to $x=0$. Since the period of the particle's motion will be $2 \Delta t_{f}$, it will be at $x=0$ whenever $t=(2 n+1) \Delta t_{f}$ and back at $x=x_{0}$ for $t=2 n \Delta t_{f}$, where $n \in\{0,1,2, \ldots\}$.

At any particular time $t$, we can solve these expressions to find the set of particles currently at the maximal heights on their trajectories; these particles are defined by $x=$ $x_{0}=g t^{2} / 8 n^{2}$. A similar procedure gives the set of particles currently at $x=0$ as $x_{0}=g t^{2} / 2(2 n+1)^{2}$. Then the region in $x_{0}$-space of initial conditions for which particles will have bounced $k$ times can be written as

$$
R_{k}=\left\{x_{0}: \frac{g t^{2}}{2(2 k+1)^{2}} \leq x_{0} \leq \frac{g t^{2}}{2(2 k-1)^{2}}, x_{0} \leq L\right\} .
$$

For any time $t>0$, there will be regions that have bounced an arbitrarily large number of times, since the bounce period goes to zero as $x_{0}$ goes to zero. However, at later 
times, there will be a minimal $k$ for which $R_{k}$ is nonempty. We can find the minimal $k$ by looking at the slowestbouncing particle, which will have $x_{0}=L$. The result is that

$$
k_{\min }=\left\lfloor\frac{1}{2}+\frac{t}{2} \sqrt{\frac{g}{2 L}}\right\rfloor .
$$

\section{From Trajectories to Density Waves}

Within each individual region $R_{k}$, we can solve for the constituent particles' trajectories explicitly. A particle that has bounced $k$ times and that started at $x=x_{0}$ at $t=0$ last bounced at $t_{\text {bounce }}=(2 k-1) \sqrt{2 x_{0} / g}$. Then until the particle leaves $R_{k}$ (that is, until its next bounce), we can write its position as

$$
x(t)=-\frac{g}{2}\left(t-t_{\text {bounce }}\right)^{2}+\sqrt{2 g x_{0}}\left(t-t_{\text {bounce }}\right) .
$$

From this expression, we would like to get a density distribution $n_{k}(x)$ for the elements of $R_{k}$, in the continuous limit (so that we imagine initially having $n_{0}$ particles per unit length). Now, in the limit as $k \rightarrow \infty$, this is an easier problem: the region $R_{k}$ becomes arbitrarily thin in $x_{0}$-space, so the particles in $R_{k}$ just trace out the trajectory of the $k$ th bounce of a particle that starts at $x_{0}$. In this case, the origin of a saw-toothed density distribution is intuitively clear, since we can just invert the velocity of the particle to get the relative density (and the velocity will be larger at small $x$ and approaches zero around $\left.x=g t^{2} / 8 k^{2}\right)$, so the density distribution will be smaller at small $x$, then monotonically increasing until it diverges around $x=g t^{2} / 8 k^{2}$.

For smaller $k$, this intuitive picture is still a good guide, but the analytical explanation is made somewhat more complicated by the fact that we must account for substantial differences in $x_{0}$ within $R_{k}$. We can begin to see this when we check the maximum value of $x(t)$ for $x_{0} \in R_{k}$; we find that $d x / d x_{0}=0$ when $x(t)=g t^{2} / 2\left(4 k^{2}-1\right)$, so the particle reaching the maximum of its own trajectory is actually not the particle currently at the largest height in $R_{k}$.

To find the density distribution corresponding to $R_{k}$, we can start by inverting our expression for $x(t)$ to get

$$
x_{0}=\left[\frac{k t \sqrt{2 g} \pm \sqrt{g t^{2} / 2-\left(4 k^{2}-1\right) x}}{4 k^{2}-1}\right]^{2} .
$$

This expression for $x_{0}$ as a function of $x$ is dual-valued, as it should be; for any non-extremal value of $x$, there is a corresponding particle with one $x_{0}$ that is moving upwards and a second particle with a different $x_{0}$ that is moving downwards.

To get $n_{k}(x)$, we want to look at the absolute value of $n_{0} d x_{0} / d x$. It is necessary to include the corresponding contributions both from the side of the distribution that is moving up and from the side of the distribution that is moving down. The result is that

$$
\begin{aligned}
n_{k}(x) & =n_{0} \frac{d}{d x}\left[\frac{k t \sqrt{2 g}-\sqrt{g t^{2} / 2-\left(4 k^{2}-1\right) x}}{4 k^{2}-1}\right]^{2} \\
& -n_{0} \frac{d}{d x}\left[\frac{k t \sqrt{2 g}+\sqrt{g t^{2} / 2-\left(4 k^{2}-1\right) x}}{4 k^{2}-1}\right]^{2} .
\end{aligned}
$$

If we evaluate these terms, the final expression becomes

$$
n_{k}(x)=\frac{4 k t n_{0} \sqrt{g}}{\left(4 k^{2}-1\right) \sqrt{g t^{2}-2\left(4 k^{2}-1\right) x}} .
$$

This expression has all of the major characteristics that we had expected. The density profile is monotonically increasing in $x$, until it diverges at $x=g t^{2} / 2\left(4 k^{2}-1\right)$, which is the maximal allowed value of $x$ for $x_{0} \in R_{k}$. If $x_{\max }$ is defined as this value, then we can rewrite the distribution function as

$$
n_{k}(x)=\frac{4 k n_{0}}{\left(4 k^{2}-1\right) \sqrt{1-x / x_{\max }}} .
$$

Of course, this expression does not take into account the requirement that $x_{0}<L$. As a result, the distribution corresponding to the $R_{k}$ with $k=k_{\text {min }}$ will have a somewhat different shape, but the distributions for all other values of $k$ will be unaffected. We can see this effect in Figure 1, where there is sometimes a small, sharp step in the density distributions in the outermost saw-tooth.

Quantitatively, the effect on $n_{k_{\min }}$ depends on whether the particle that started at $x_{0}=L$ is on its way up or on its way down - that is, if $L$ is greater than or less than

$$
x_{0, \max }=\frac{2 g k^{2} t^{2}}{\left(4 k^{2}-1\right)^{2}},
$$

which is the value of $x_{0}$ from which a particle has to start in order to end up at $x_{\max }$ at time $t$. This tells us if $x_{0}=L$ is on the upward-moving or the downward-moving branch of $x_{0}(x)$.

Then we can define the critical value of $x$ corresponding to the height of the particle that started at $x_{0}=L$, that is,

$$
x_{\mathrm{crit}}=-\frac{g t^{2}}{2}+2 k t \sqrt{2 g L}+\left(1-4 k^{2}\right) L .
$$

There are a few possible scenarios. If $x$ and $t$ are such that both the upward- and downward-moving branches of $x_{0}(x)$ have $x_{0}<L$, then $n_{k_{\text {min }}}(x)$ is as previously calculated; we will call this original distribution function $n_{k}^{(0)}$. If $x$ and $t$ are such that the downward-moving branch has been cut off by the boundary condition $x_{0}<L$, then we keep only the first of the two terms in Equation 5, and the result is

$$
n_{k}^{(1)}=\frac{2 k n_{0}}{\left(4 k^{2}-1\right) \sqrt{1-x / x_{\max }}}-\frac{n_{0}}{4 k^{2}-1} .
$$



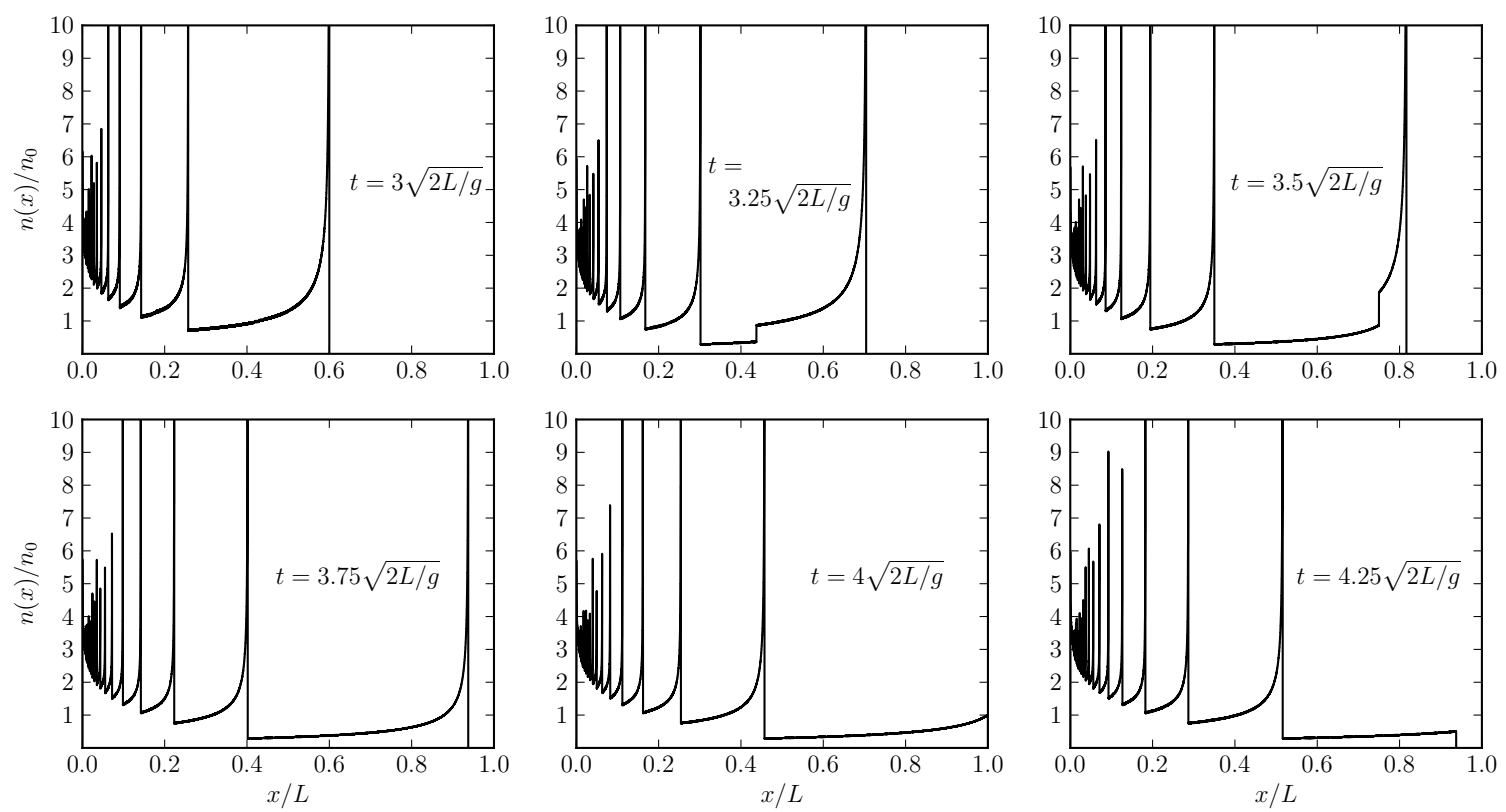

Figure 1: These plots show snapshots of the density distribution with varying times. To get these distributions, we computed the positions of one million particles that started out uniformly distributed from 0 to $L$ at $t=0$.

Finally, if both branches that would contribute $n_{k_{\min }}$ at $x$ are cut off by the boundary condition, then the density distribution at $x$ must go to 0 . In general, then, the outermost wave has a density distribution governed by

$$
n_{k_{\min }}(x)= \begin{cases}n_{k}^{(0)}(x) & : x_{0, \max }<L \text { and } x>x_{\mathrm{crit}} \\ n_{k}^{(1)}(x) & : x<x_{\mathrm{crit}} \\ 0 & : x_{0, \max }>L \text { and } x>x_{\mathrm{crit}}\end{cases}
$$

\section{Density Wave Structure}

The total density distribution of the system is given by

$$
n(x)=\sum_{k=k_{\min }}^{\infty} n_{k}(x) .
$$

That is, the overall distribution is a sum of infinitely many sawtooth-like waves. However, for any given $x$, the sawtooth corresponding to $R_{k}$ only contributes to $n(x)$ if $x \leq$ $g t^{2} / 2\left(4 k^{2}-1\right)$. For instance, at $t=\sqrt{2 L / g}, n(L / 20)$ only includes contributions from $n_{1}(x)$ and $n_{2}(x)$. At $t=2 \sqrt{2 L / g}, n(L / 20)$ includes contributions from $n_{1}(x)$, $n_{2}(x), n_{3}(x)$, and $n_{4}(x)$.

If we change coordinates to use the normalized position $\bar{x}=x / L$, then the system parameters $g$ and $L$ only ever appear in the dynamics of the system in the combination $g t^{2} / L$. Then if we define $\tau=t \sqrt{g / L}$, the density profile $n$ can be written as a function only of $\bar{x}$ and $\tau$.
As such, the system is invariant under any transformation that preserves $\tau$, such as simultaneously mapping $g \rightarrow \lambda g$ and $L \rightarrow \lambda L$. Any modification of the system parameters $g$ and $L$ only has the effect of speeding up or slowing down the evolution of $n(\bar{x}, t)$ by a constant factor. In light of this, Figure 1 could be interpreted as the time evolution of the system for fixed $g$ and $L$, but it also shows the system's behavior when $g$ or $L$ are varied at some fixed time.

We can see from our expressions for $x_{\max }$ and $n_{k}(x)$ that these density waves will originate at $x=0$ and move to larger $x$ until they disappear at $x=L$. The dependence of $n_{k}(x)$ on $k$ shows that the waves will appear thinner as time goes on, although defining a wave amplitude is tricky given that every wave peak is infinitely tall. There is always an infinite number of wave peaks in $0 \leq x \leq L$, but for any region $0<\epsilon \leq x \leq L$, there will be an increasing number of wave peaks as time goes on and the peaks will get closer together. We can see this by looking at the peakto-peak separation in $x$; the distance between the $k$ th and $(k+1)$ th peak is given by

$$
\begin{aligned}
\Delta x_{k, k+1} & =\frac{2 g t^{2}}{(2 k-1)\left(4 k^{2}+8 k+3\right)} \\
& =\left(\frac{4}{2 k+3}\right) x_{\max , k} .
\end{aligned}
$$

Here $x_{\max , k}$ is the location of the $k$ th peak. As each successive peak reaches any given height $x$, the distance to the next one decreases and will scale like $1 / k$ for long times.

Our expression for $k$ th peak's location also allows us 

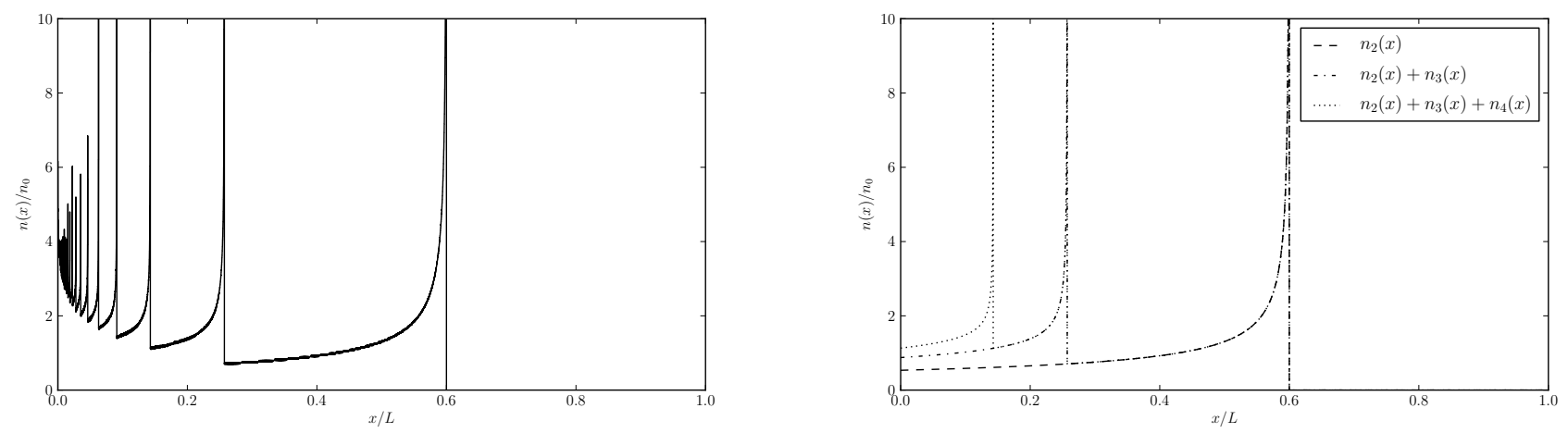

Figure 2: The left plot shows a density distribution at $t=3 \sqrt{2 L / g}$ obtained by computing the trajectories of one million particles. The right plot shows the density distributions corresponding to our analytical result for the first few nonempty $R_{k}$ intervals.

to compute that the velocity of the $k$ th peak is given by

$$
v_{k}=\frac{g t}{4 k^{2}-1} .
$$

All of the peaks move more quickly as time goes on, but the ratio between the velocities of any two peaks' velocities does not change. At any given time, higher- $k$ peaks move more slowly. Moreover, the value of $k$ corresponding to the peak at a particular height is roughly linear in time (it is linear for long times); the velocities of wave peaks passing any particular height will decrease as time goes on and will eventually scale like $1 / t$.

For long times, the details of these wave structures become less and less obvious. We can analytically compute the shape of the final distribution $n(x)$ in the limit that the particles starting from different initial conditions are completely uncorrelated. For any fixed $x_{0}$, we can invert $x(t)$ to find that the amount of time the particle spends at a position scales like $\left(x_{0}-x\right)^{-1 / 2}$. If we account for the different period lengths at different values of $x_{0}$, then $n(x)$ for a fixed $x_{0}$ is proportional to $\left(x_{0}^{2}-x x_{0}\right)^{-1 / 2}$. Then accounting for the whole distribution of values of $x_{0}$, and considering the fact that a particle only contributes to the distribution at $x$ if it has $x_{0} \geq x$, we get

$$
n(x) \propto \int_{x}^{L} \frac{d x_{0}}{\sqrt{x_{0}^{2}-x x_{0}}} .
$$

Integrating and normalizing, the result is that

$$
n(x)=n_{0} \log \left(\frac{\sqrt{L}+\sqrt{L-x}}{\sqrt{x}}\right) .
$$

In fact, it is possible to recover precisely this result by looking at the sum over $n_{k}(x)$ for large $t$. For sufficiently large time, we can write that $k \rightarrow(t / 2) \sqrt{g / 2 x_{\max }}$, and for a given $x$, the terms in the sum over $n_{k}$ that contribute are given by

$$
n(x)=\int_{(t / 2) \sqrt{g / 2 L}}^{(t / 2) \sqrt{g / 2 x}} \frac{4 k n_{0} d k}{\left(4 k^{2}-1\right) \sqrt{1-x / x_{\max }}} .
$$

Changing variables and noting that $4 k^{2}-1 \rightarrow 4 k^{2}$ for sufficiently large $t$,

$$
\begin{aligned}
n(x) & =\int_{x}^{L} \frac{n_{0} d x_{\max }}{2 x_{\max } \sqrt{1-x / x_{\max }}} \\
& =n_{0} \log \left(\frac{\sqrt{L}+\sqrt{L-x}}{\sqrt{x}}\right) .
\end{aligned}
$$

\section{Possible Applications}

Beyond the intrinsic interest of the dynamics of such a system, these density waves could have some practical applications. If we could produce a system with the behavior described here, we could use that system to measure the value of $g$. Such a detector might not easily compete with the precision that can be achieved with measurements by atomic interferometry [13-15], using the properties of cold atoms [16], or other modern methods, but it might have some advantages in simplicity.

Consider a container filled with particles that are diffuse enough that their mean free path is at least the height of the container, with the property that they bounce elastically when they strike the surfaces of the container. If the particles were released into the container with a uniform density distribution in height and with sufficiently small initial velocities, and if the container were equipped with instrumentation to measure the density of the particles as a function of height, then it would be possible to determine $g$ by measuring the locations of the wave peaks at $x(t)=g t^{2} / 2\left(4 k^{2}-1\right)$.

Of course, any kinematic scheme for measuring gravitational fields is only interesting if it can outperform the simplest possible kinematic $g$ measurement device: a detector consisting of a bouncing ball and a device that measures the length of its bounce periods (or its position at a time $t$ ). Such a detector would lack the practical challenge of preventing collisions between particles.

These density waves have a couple of advantages. The first has to do with statistical fluctuations. The density 
waves' behavior depends on a whole ensemble of particles, so measurements of the wave peaks' positions would be resistant to some sources of error (for instance, variations in the initial particles' initial positions). In principle, we could also set up an ensemble of objects to be dropped from the same height, but if the collision rate of the particles scales with their local density, then this would result in a higher collision rate for the same number of particles.

The second major advantage is that the peaks of the waves propagate more slowly than their constituent particles move, and many wave peaks will be visible at the same time; if the location of each wave peak provides another measurement of $g$, then it may be possible to get statistical precision more quickly under some conditions (since we can take effectively take many measurements of $g$ at the same time).

There are some cases in which the density wave system easily produces better measurements of $g$. For instance, for any system size, it is possible to pick a time interval between measurements that makes position measurements of the single-bouncing-ball system virtually useless; if the time between measurements is comparable to the period of the bounce, then we could no longer tell how many times the ball has bounced. The same is not true of the densitywave system, because (with sufficiently precise position measurements) we can always identify the $k$ th wave peak by measuring the inter-peak distances and using Equation 13. On the other hand, there are also situations in which the single-bouncing-ball system is definitively better. For instance, if the spatial resolution is not sufficient to resolve the spacing between peaks, then the density wave structures cease to be useful. If we wait a sufficiently long time, this will happen for any finite spatial measurement precision.

In less extreme cases, the effects of measurement uncertainty are more complicated. Generally speaking, the single-bouncing-ball model is more robust against uncertainty in spatial position, whereas the density-wave model is more robust against uncertainty in time. As a heuristic model, consider a system in which we have perfect initial conditions (perhaps because of the statistical benefits of a large sample size) and perfect spatial measurements but imperfect temporal measurements, so that measurements of the density distributions will be characterized by some standard deviation $\sigma_{t}$. We will compare this against a model in which we drop a bouncing ball from some height $h$, again with perfectly precise initial conditions and perfectly precise position measurements, and measure its position at different times with the same precision $\sigma_{t}$ (in either case, one might imagine watching the system evolve and trying to track with a stop-watch how long it takes to reach certain fixed points).

Consider a measurement taken at some time $t$. For the density-wave system, the location of the $k$ th peak is given by $x=g t^{2} / 2\left(4 k^{2}-1\right)$. Then we can estimate the error on an estimate of $g$ given an imperfect measurement of $t$ by

$$
\begin{aligned}
\sigma_{g, k} & =\left|\frac{\partial g}{\partial t}\right| \sigma_{t} \\
& =\frac{4\left(4 k^{2}-1\right) x \sigma_{t}}{t^{3}} .
\end{aligned}
$$

For our overall estimate of $g$, we will want to combine the information from several peaks, each of which will give us a different level of precision for $g$. In order to combine such measurements, we would take the weighted average of the measurements, where the weight of each peak's contribution is given by $1 / \sigma_{g, k}^{2}$ for that peak [17]. The overall error would then be given by

$$
\begin{aligned}
\sigma_{g} & =\sqrt{\frac{1}{\sum_{k} 1 / \sigma_{g, k}^{2}}} \\
& =\left[\sum_{k=k_{\min }}^{k_{\max }} \frac{1}{\left(4 k^{2}-1\right)^{2} x^{2}}\right]^{-1 / 2} \frac{4 \sigma_{t}}{t^{3}} .
\end{aligned}
$$

The lower limit of this sum is the same $k_{\text {min }}$ from Equation 2 , that is, $k_{\min }=\lfloor 1 / 2+(t / 2) \sqrt{g / 2 L}\rfloor$. The upper limit will be the largest $k$ for which the $k$ th wave peak can be distinguished from the $(k+1)$ th peak. Using Equation 13, this means that

$$
\frac{2 g t^{2}}{\left(2 k_{\max }-1\right)\left(4 k_{\max }^{2}+8 k_{\max }+3\right)} \approx \sigma_{x} .
$$

This starts getting messy quickly. However, once we know $k_{\min }$ and $k_{\max }$ for a particular choice of parameters, then we can simplify our expression for $\sigma_{g}$ by writing that $k_{\max }-$ $k_{\text {min }}$ is the total number of discernible peaks and substituting in our expression for the peak position to get

$$
\sigma_{g}=\frac{2 g \sigma_{t}}{t \sqrt{k_{\max }-k_{\min }}} .
$$

The quantity $k_{\max }-k_{\min }$ is still somewhat complicated. The distance between the $k$ th and $(k+1)$ th peak is quadratic in time, but each pair of peaks has a maximal separation that it will achieve before they disappear, and any peak that is currently possible to resolve will disappear in a finite time. If the resolution is small compared to the system size, this means that the number of discernible peaks will start at some finite value, then increase for a period of time, then decrease to zero.

The version with a single bouncing ball only provides a single measurement at any time $t$. If we use the same procedure to estimate $\sigma_{g}$ for this measurement, then if the ball was dropped from a height $h$ and has bounced $b$ times, we get

$$
\begin{aligned}
\sigma_{g} & =2(2 b \sqrt{2 h} \pm \sqrt{2(h-x)})^{2} \frac{\sigma_{t}}{t^{3}} \\
& =\frac{2 g \sigma_{t}}{t}
\end{aligned}
$$


This function is double-valued because for any given measurement of $x$ there are two possible choices of $g$, since the ball reaches that height twice on every bounce. We will assume for now that our measurements are frequent and precise enough that we can tell when the ball is moving up, when it is moving down, and how many times it has bounced, so that these are not additional sources of error. Under these assumptions, the measurements of $g$ from the density-wave model are more robust against errors in time measurement so long as we can reliably identify more than one wave peak at a time.

An alternate possible approach by which these density waves could be used to measure $g$ involves introducing a perturbation to the potential. In particular, if we could introduce a sinusoidal time-varying perturbation with an appropriately chosen phase, we could set up a stable limit cycle, which would result in a significant number of particles oscillating together at a particular frequency. For instance, consider the perturbation $g \rightarrow g-r g \cos (\omega t)$ for some $0<r<1$. For this perturbation, there are stable limit cycles at the trajectories of the particles starting at $x_{0}=2 g \pi^{2} n / \omega$ for $n \in\{1,2, \ldots\}$. Trajectories with $x_{0}$ slightly above these limit cycles will experience, on average, higher values of $g$ as they initially fall, while trajectories with $x_{0}$ slightly below will initially experience lower values of $g$. As such, for any driving frequency $\omega$, we could measure $g$ by looking for long-lived oscillations in the density profile with frequencies equal to $\omega / n$ that go between $x=0$ and $x=2 g \pi^{2} n / \omega$. We might introduce such a perturbation to $g$ by shaking the system back and forth at the given frequency.

This approach has the advantage that it doesn't require particularly precise initial conditions; there could be some variation in the initial density and the limit cycles would still produce observable and distinctive effects. It also has the advantage that the limit cycle will stay where it is indefinitely. In the unperturbed version of the system, the density waves get closer together with time until they eventually become impossible to resolve, but this version does not have that problem. The robustness of this system to measurement uncertainty is basically comparable to that of the bouncing-ball system, but this system has the advantage that it does not require any minimal sampling rate, so long as we could identify the maximal height that each pulse reaches.

Either of these techniques could also be applied to a system of charged particles in a constant electric field, to produce measurements of $\vec{E}$. However, such a detector would be less practical, since it would be substantially harder to prevent the inter-particle interactions. This would likely force the starting density of the particles to be much lower, so the system would have to occupy a much larger volume in order to achieve the same statistics.

\section{Discussion}

We initially encountered these density waves in a series of simulations modeling particles in simple potentials which were motivated by another problem (which involved temperature gradients that can form in gases as they are compressed in an external potential [18]). This, in turn, was motivated by an investigation into the physics of rotating plasmas and gases $[19,20]$.

In fact, our results are somewhat reminiscent of a phenomenon from plasma physics: the plasma wave echo [21]. In a plasma echo, two waves that damp in a collisionless plasma can reappear (as an "echo") some time after they may seem to be gone. Plasma echoes are counterintuitive because we would ordinarily expect phase space mixing to wash out the initial characteristics of the system, but it does not do so completely. The result in this paper is similar; we have described a projection of phase space as a function of time which does not reach a smooth, statistically averaged final state in the way that we might expect.

The system that we studied contained no inter-particle interactions. For the purposes of translating these results to a practically realizable system, this is a drawback. Simple numerical simulations do suggest that for sufficiently weak inter-particle interactions and over sufficiently short timescales, these density wave structures can still be prominent. However, if (for a given model of interparticle interactions and over a given timescale) these density waves are important, it is in spite of these interactions rather than because of them.

In our view, the lack of inter-particle interactions is a large part of what makes this density wave effect interesting. These saw-toothed structures look very much like some kind of collective effect, but each individual particle's dynamics are completely independent of the rest. This happens in an almost superlatively simple physical system, and without the need for any kind of fine-tuned initial density distribution.

\section{Conclusions}

Using a very simple system of bouncing particles and completely smooth initial conditions, we have shown the emergence of a surprisingly complex pattern of infinitely sharp density waves. The most important factor that contributes to this effect is the reflecting lower boundary. In fact, we find similar density wave structures for a variety of different choices of potential (although the details of the behavior of these waves do change with different potentials; for instance, it is possible to choose potentials so that the waves propagate from high $x$ to low $x$ rather than the other way around, or even so that the waves propagate in one direction in some regions and in the other in other regions).

If we could construct a physical system with the general characteristics described by this model, these density 
waves could be used to measure the strength of the underlying potential. There are a couple of different possible ways of doing this, with different advantages and disadvantages, but the most basic version of the system is notable in that it is robust against uncertainty in time measurements and in that it does not require any minimal sampling rate.

\section{Acknowledgments}

This work was supported by the U.S. Defense Threat Reduction Agency, and by the NNSA SSAA Program through DOE Research Grant No. DE-FG52-08NA28553.

[1] T. M. Mello and N. B. Tufillaro, Am. J. Phys. 55, 316 (1987).

[2] Z. J. Kowalik, M. Franaszek, and P. Pierański, Phys. Rev. A 37, 4016 (1988).

[3] T. Kroetz, A. L. P. Livorati, E. D. Leonel, and I. L. Caldas, Phys. Rev. E 92, 012905 (2015).

[4] A. L. P. Livorati, T. Kroetz, C. P. Dettmann, I. L. Caldas, and E. D. Leonel, Phys. Rev. E 86, 036203 (2012).

[5] E. D. Leonel and M. R. Silva, J. Phys. A 41, 015104 (2008).

[6] F. Saif, I. Bialynicki-Birula, M. Fortunato, and W. P. Schleich, Phys. Rev. A 58, 4779 (1998).

[7] A. J. Lichtenberg and M. A. Lieberman, Regular and Stochastic Motion, Applied Mathematical Sciences, Vol. 38 (SpringerVerlag, 1983).

[8] P. J. Holmes, J. Sound Vib. 84, 173 (1982).

[9] J. M. Luck and A. Mehta, Phys. Rev. E 48, 3988 (1993).

[10] M. F. Andersen, A. Kaplan, N. Friedman, and N. Davidson, J. Phys. B 35, 2183 (2002).

[11] S. H. Strogatz, Nonlinear Dynamics and Chaos, 2nd ed. (Westview Press, 2015).

[12] G. X. Li, R. H. Rand, and F. C. Moon, Int. J. Non-Linear Mechanics 25, 417 (1990).

[13] J. B. Fixler, G. T. Foster, J. M. McGuirk, and M. A. Kasevich, Science 315, 74 (2007).

[14] J. M. McGuirk, G. T. Foster, J. B. Fixler, M. J. Snadden, and M. A. Kasevich, Phys. Rev. A 65, 033608 (2002).

[15] A. Peters, K. Y. Chung, and S. Chu, Metrologia 38, 25 (2001).

[16] S. Schlamminger, Nature 510, 478 (2014).

[17] J. R. Taylor, An Introduction to Error Analysis (University Science Books, Sausalito, CA, 1997)

[18] V. I. Geyko and N. J. Fisch, " arXiv:1604.00063.

[19] V. I. Geyko and N. J. Fisch, Phys. Rev. E 90, 022139 (2014).

[20] V. I. Geyko and N. J. Fisch, Phys. Rev. Lett. 110, 150604 (2013).

[21] R. W. Gould, T. M. O'Neil, and J. H. Malmberg, Phys. Rev. Lett. 19, 219 (1967). 